\title{
Apprendre à parler en langue régionale pour un devenir citoyen
}

\section{Philippe Martel}

\section{OpenEdition}

1 Journals

\section{Édition électronique}

URL : http://journals.openedition.org/trema/1752

DOI : 10.4000/trema.1752

ISSN : 2107-0997

\section{Éditeur}

Faculté d'Éducation de l'université de Montpellier

\section{Édition imprimée}

Date de publication : 1 octobre 1999

Pagination : 135-145

ISSN : 1167-315X

\section{Référence électronique}

Philippe Martel, «Apprendre à parler en langue régionale pour un devenir citoyen », Tréma [En ligne], 15-16 | 1999, mis en ligne le 01 octobre 1999, consulté le 07 mai 2019. URL : http:// journals.openedition.org/trema/1752 ; DOI : 10.4000/trema.1752

Ce document a été généré automatiquement le 7 mai 2019.

Trema 


\title{
Apprendre à parler en langue régionale pour un devenir citoyen
}

\author{
Philippe Martel
}

\section{Présentation de l'atelier. Philippe MARTEL}

1 Le titre donné à cet atelier pourra surprendre, au premier abord. Langue régionale (l'occitan ou le catalan par exemple), devenir, citoyenneté : trois mots que l'on n'est guère habitué à associer en France. Du fait, d'abord, d'un long héritage idéologique, qui plonge ses racines dans la culture d'Ancien Régime, celle qui opposait la langue de l'honnête homme, d'autant plus honnête qu'il était plus proche du Roi, et le "patois » abandonné à la populace, comme on disait du temps de l'Encyclopédie. En d'autres termes, il y avait une langue qui pouvait tout dire, le Beau, le Vrai en premier lieu, et des idiomes qui en étaient bien incapables. Cette idée a survécu au Roi et à sa Cour, pour être reprise par leurs vainqueurs, puisqu'aussi bien ils étaient au fond, de purs produits, quoique ô combien déviants, de cette culture des élites, qui demeure la même, quel que soit le profil exact des élites en question. Un brave savetier tarnais pouvait bien, au fond de sa campagne, inventer dans une chanson de 1792 en occitan le personnage de Marianne, la future personnification de la République (mais qui le sait, aujourd'hui, en dehors des lecteurs de Maurice Agulhon?), l'affaire n'en était pas moins entendue: puisque le fanatisme parlait basque et la superstition bas-breton, la Révolution ne pouvait certes pas parler patois. Les mentalités n'ont assurément pas beaucoup bougé depuis. Et l'opinion s'est somme toute habituée à l'idée que ces idiomes ruraux devaient suivre dans la tombe les derniers vestiges des campagnes d'autrefois, au fur et à mesure que la modernité progressait dans nos provinces.

2 Cette mort ne suscitait d'ailleurs pas nécessairement des sentiments d'exultation. De bons esprits pouvaient - peuvent encore la déplorer, tout en l'attribuant à une sorte de fatalité incontournable, ou de nécessité évolutive supérieure. On ne fait pas d'omelette sans casser des œufs, avons-nous pu entendre : pour faire la France, il a fallu casser deux œufs, l'Occitanie et le Protestantisme, (sic)... Mais l'omelette est si bonne! Certains peuvent 
même pousser la déploration au delà de cette résignation patriotique distraite, et célébrer, dans le droit fil d'une tradition chérie par les livres de lecture de la Troisième République, le souvenir nostalgique de ce bon vieux temps des champs cultivés et des fermes actives, le temps des racines et du verbe savoureux de nos terroirs. Pour ceux-là, il $\mathrm{y}$ a, certes, une place pour les langues régionales, au magasin des antiquités nationales, comme ornement, malheureusement virtuel, de ces écomusées qui poussent de nos jours comme des champignons dans les zones reculées où l'activité humaine recule. Mais on voit bien que cette place est limitée: ces langues sont d'hier: elles ne sauraient dire demain, et du coup, à quoi bon les enseigner aujourd'hui ? Ne vaut-il pas mieux les laisser à la disposition des amateurs de vieilles choses, et fournir à nos enfants un kit culturel bien mieux adapté à la modernité telle qu'elle se décline?

Ces deux positions se rejoignent sur un point : elles excluent toute possibilité de survie pour l'occitan, ou le catalan - celui de France s'entend, car on commence à savoir, quitte à le déplorer, qu'il ne se porte pas si mal en Espagne - mais c'est si loin! On croit volontiers en France que ces langues sont déjà mortes, ou tout au plus encore balbutiées par quelques vieillards. Qu'en feraient nos enfants ? Il est de toute façon trop tard.

puis il y a ceux qui n'éprouvent ni nostalgie ni regret, et perçoivent au contraire comme une menace toute action en faveur d'idiomes perçus comme politiquement dangereux pour la cohésion de la Nation. Se revendiquer Occitan ou Breton, n'est-ce pas se couper de la communauté nationale, qui a plus que jamais besoin d'affirmer son unité, au moment où elle est menacée simultanément par la fracture sociale qui exclut de fait des citoyens de plus en plus nombreux, et par la construction européenne qui mine les fondements de l'Etat Nation le plus ancien de la galaxie? Au pire, cette quête de l'identité linguistique perdue ne peut-elle faire le jeu des partisans de la fermeture absolue, de ceux qui se replient sur les totems ancestraux pour mieux se protéger du voisinage inquiétant de l'Autre? Idiomes du passé, les parlers régionaux ne sont ils pas, par excellence, vecteurs de passéisme?

On voit bien que dans ce contexte, parler de l'apport de l'enseignement des langues régionales dans l'enseignement public à la construction d'une nouvelle citoyenneté peut relever au mieux de la gageure, au pire de l'escroquerie. Et pourtant, c'est bel et bien de cela qu'il a été question dans cet atelier. La dizaine de personnes qui s'y est retrouvée n'a pas cherché à répondre point par point à toutes les objections que nous venons de résumer. L'atelier n'a pas davantage cherché à élaborer une défense et illustration de l'occitan ou du catalan, drapeaux au vent. Si le même jour une manifestation pour l'occitan se déroulait à Montpellier - nous privant du même coup d'une partie de notre public potentiel, le ton, parmi ceux qui étaient venus à l'IUFM, se voulait moins vigoureusement militant. Il s'agissait davantage de réfléchir, à partir d'expériences concrètes, sur ce que pouvait apporter à l'enfant l'apprentissage d'une langue régionale. Non point en termes d'identité - c'est le sujet qui se bâtit sa propre identité, quitte à la nourrir, à la marge, de ce que lui apporte l'école - et nous ne croyons guère au concept d'identité collective. Mais en termes de savoirs, de savoir-parler, de savoir-lire, de savoirpenser le monde qui l'entoure.

6 Les participants constituaient un échantillon assez représentatif: on y trouvait des enseignants consacrant à l'occitan, ou au catalan l'essentiel de leur activité, soit à l'IUFM, soit à l'Université, soit encore dans un établissement secondaire, puisqu'il existe aujourd'hui des certifiés d'occitan. Mais on y trouvait aussi des maîtres du primaire, qui, par force, ne pouvaient consacrer à l'occitan qu'une partie de leur enseignement, compte 
tenu de la grande misère de l'enseignement bilingue dans les écoles primaires de notre région. On y trouvait enfin de futurs enseignants - ou enseignantes, au cas particulier, séduites par l'idée d'enseigner la langue régionale, et venues voir comment cela pouvait se faire. Il manquait une composante, toutefois: des représentants de cette grande majorité qui ne sait rien de la question de l'enseignement des langues régionales, ne se sent absolument pas concernée par elle, ignorait même jusque là qu'elle pouvait se poser, et n'avait en tête dans le meilleur des cas que l'ombre des préjugés dont nous parlions plus haut. Ceux-là n'auront su du déroulement de notre atelier que ce qui en aura été répercuté en séance plénière, c'est-à-dire fort peu de choses. Si fructueux qu'ait pu être l'échange entre les personnes présentes, déjà convaincues pour l'essentiel, elles auraient eu besoin du contrepoint que seuls peuvent fournir des observateurs étrangers au problème.

7 La parole a d'abord été donnée à des praticiens de l'enseignement de l'occitan, officiant à deux niveaux différents, l'un dans le primaire, l'autre dans le secondaire - et, depuis peu, dans le Supérieur.

8 Francis Escudier est conseiller pédagogique pour l'enseignement de l'occitan dans le primaire, et il donne par ailleurs des cours à l'IUFM. Son point de vue est donc à la fois celui d'un homme de terrain, qui a eu l'occasion de voir fonctionner concrètement l'occitan dans les classes de l'Hérault, et celui d'un homme qui a les moyens de prendre un peu de recul par rapport à ses observations empiriques. Son témoignage est donc en même temps un point de vue. Nous lui laissons la parole.

\section{Témoignage et point de vue. Francis ESCUDIER}

Il n'est qu'à énumérer les multiples difficultés administratives auxquelles est confronté l'enseignement de l'occitan dans le cadre de l'enseignement public pour se conforter dans l'idée que cet enseignement est un enjeu politique qui débouche à terme sur un choix de société.

Mais ne serait-il pas illusoire de croire que la volonté de généraliser l'enseignement précoce des langues étrangères, avec la dominance incontestable de l'anglais, ne procède pas, elle aussi, d'un choix politique qui induit ou avalise un choix de société?

La question linguistique n'est qu'un voile qui cache d'autres enjeux, politiques et culturels.

En effet, la culture est première, et, sans jeu de mots, la langue est seconde.

Ceci étant posé, la question est de savoir en quoi l'enseignement de la langue et de la culture régionales, et donc la connaissance par les enfants de cette langue et de cette culture contribue à la formation du futur citoyen, et, ajouterai-je, lequel ?

11 Je vais donc essayer d'apporter quelques éléments qui pourront alimenter notre réflexion, et par là-même, le débat.

Le premier jour où un enfant entre à l'école maternelle, l'enseignant lui fait généralement visiter les lieux où il va vivre pendant quelquefois plus de 8 heures par jour.

Pourquoi ? Tout simplement parce que l'enfant a d'abord besoin d'être sécurisé, il doit se familiariser avec son environnement.

12 L'enseignant agit ainsi afin de donner quelques repères spatiaux à l'enfant qui ainsi pourra mieux se mouvoir, vivre et agir dans ce cadre nouveau pour lui. 
Mais l'enfant a besoin d'autres repères, toujours très proches de lui, appartenant à son cercle de vie.

- Des repères temporels (heure des mamans, de la récréation, du goûter, des toilettes, du conte, etc).

- Des repères affectifs et sociaux par rapport à ses parents, ses enseignants, ses camarades. L'enfant devra situer chacun d'eux à sa bonne place.

Ecole, maison, rue, quartier, sont autant de lieux avec des us et coutumes, en quelque sorte, mais aussi avec une langue.

Tous ces repères sont indispensables, et contribuent à la structuration de l'enfant. Leur intégration au fil du temps et de proche en proche lui permet de mieux prendre possession d'un environnement de plus en plus lointain.

A une autre échelle, l'adulte ne fonctionne pas autrement, il doit lui aussi s'appuyer sur un certain nombre de repères géographiques, économiques, linguistiques, culturels, affectifs et sociaux qu'il a stratifiés et qui lui permettent de se mouvoir et d'agir dans et sur la société dans laquelle il vit.

L'absence de repères, leur ignorance ou leur perte, mène à une perte d'autonomie et donc de liberté. Cela peut le conduire jusqu'à l'exclusion.

Or nous vivons dans une société où justement ces ancrages structurants sont ignorés, méprisés sinon volontairement détruits au profit de repères étrangers à l'individu, de plus en plus lointains. Les repères de proximité sont évacués au profit de repères supranationaux inaccessibles à la plupart des citoyens (mais le sont-ils encore ?) qui perdent ainsi la maîtrise de leur environnement et donc la liberté d'agir, de peser sur la société. Son fonctionnement leur échappe, car les stratégies économiques ignorent les hommes sauf bien sûr à les utiliser à leurs propres fins.

Ainsi l'occultation de certains repères de proximité mène à une forme d'acculturation du citoyen qui ne peut donc véritablement exercer sa citoyenneté. C'est l'exclusion citoyenne.

C'est justement ici que l'enseignement de la langue et de la culture régionales contribue à la formation du futur citoyen, car à son niveau, cet enseignement s'efforce précisément de faire prendre conscience aux enfants des divers éléments de langue et de culture qui appartiennent à leur vécu de chaque jour et qui sont autant de repères de proximité dont le champ bien sûr s'élargira peu à peu au cours de leur scolarité.

Si l'on veut traverser une rivière à gué, toutes les pierres doivent être en place. Essayez donc de supprimer les premières !

La langue régionale fait partie intégrante de cette démarche. Linguistiquement proche du français, pour ce qui concerne l'occitan et le catalan, et en adéquation avec la culture vécue des enfants, sa connaissance permet de jeter un pont entre le français et les langues étrangères, à commencer par les langues romanes.

En début d'intervention, je parlais de l'éloignement des repères et de leur perte. La politique du «tout anglais» appliquée aux jeunes participe de ce fonctionnement. L'anglais est sans doute nécessaire dans le sens de l'adaptabilité à la société qui nous est proposée aujourd'hui. Mais quand il s'agit de donner aux enfants les bases qui permettent l'exercice d'une véritable citoyenneté, active et responsable du niveau local jusqu'au niveau européen, la connaissance de la langue et de la culture régionales est un chaînon, un ancrage, un repère tout aussi nécessaire. 
Je terminerai par une autre image que celle du gué.

Pour apprendre à nager, on sait que l'enfant doit passer par un certain nombre d'étapes. Il fut un temps où une méthode consistait à lancer directement l'enfant dans l'eau : ainsi, il était censé apprendre à se débrouiller.

Résultat: nombre d'enfants traumatisés eurent à vie une peur panique de l'eau, et à sa vue se recroquevillaient dans leur coin.

La connaissance de la langue et de la culture régionales, pour les raisons que je viens de développer, est une des étapes qui doit permettre de mieux nager (ou surfer!) dans le grand bain de l'Europe et de la mondialisation.

Sinon, je crains fort que certains, à l'âge adulte, ne se recroquevillent frileusement dans leur coin de Provence ou d'ailleurs.

La connaissance de la langue et de la culture régionales contribue à la formation du futur citoyen dans le sens de l'humanisme et non de l'économisme.

De la même manière que l'enfant doit être au centre du système éducatif, l'homme doit être au centre de la société.

(Philippe MARTEL) Après le primaire, le secondaire, abordé par Marie-Jeanne Verny, forte d'une expérience de plusieurs années d'enseignement de l'occitan dans un lycée nîmois, forte aussi de la connaissance générale de la situation de l'occitan dans le secondaire qu'elle doit à ses responsabilités au sein du CREO (Centre Régional de l'Enseignement de l'Occitan) l'association qui regroupe les enseignants concernés.

\section{Marie-Jeanne VERNY}

La place tenue par l'occitan dans les collèges et les lycées est plus facile à appréhender que celle qu'il tient dans le primaire, dans la mesure où les cursus et les programmes lui attribuent un espace plus formellement balisé. Certes, la place laissée à l'enseignement bilingue, ou du moins à un rôle de l'occitan comme langue enseignante et pas seulement enseignée, demeure dans notre académie plus que minime. Une collègue, dans un collège de Montpellier, mène depuis plusieurs années une expérience particulière : à côté de ses cours d'occitan, elle enseigne l'histoire et la géographie dans cette langue. Initialement prévu pour permettre l'accueil dans le public d'élèves issus des écoles associatives Calandretas, il s'est révélé au fil des ans que cet enseignement partiellement bilingue pouvait attirer d'autres élèves. Mais il s'agit pour l'heure, répétons-le, d'une expérience ponctuelle. Pour l'essentiel, la place de l'occitan dans les collèges est celle d'une option légère - une heure hebdomadaire pour les élèves volontaires. Par la suite, l'occitan peut intervenir comme langue vivante 2 ou 3 - à raison de 3 heures hebdomadaires, ou comme matière optionnelle facultative, en lycée, dans la perspective de l'épreuve orale facultative instituée il y a près de cinquante ans par la Loi Deixonne.

21 Dans ce cadre, l'enseignement de l'occitan, s'il se limite à son propre champ, ne peut qu'être marginal. Mais bien souvent, le travail de l'enseignant d'occitan ou de catalan l'amène à élargir cet espace. Le maître mot est ici celui d'interdisciplinarité. Il se trouve que l'enseignant d'occitan est aussi, par fonction, formé à une autre discipline. Soit qu'il s'agisse d'un enseignant donnant des cours d'occitan à côté de sa matière principale : ce fut longtemps le seul chemin, en l'absence d'une filière de recrutement spécifique. Depuis 1992, cette filière existe, sanctionnée par un CAPES. Mais ce CAPES est bivalent. En d'autres termes, le certifié d'occitan enseigne aussi, selon le cas, le français, l'histoire- 
géographie, ou une langue vivante étrangère. Du coup, la mise en place de passerelles entre disciplines est non seulement possible, mais presque incontournable.

Dans le domaine littéraire par exemple, la connaissance de la littérature française peut être enrichie par la confrontation avec la littérature d'oc. Il est difficile - bien que cela ait longtemps été pratiqué dans la plus heureuse bonne conscience - d'aborder le Moyen Age et sa littérature sans faire référence, autrement que de façon fugitive, au texte des Troubadours, puisque la lyrique d'oc a rayonné, au XII ${ }^{e}$ siècle, sur l'ensemble des littératures européennes. Plus tard, la littérature française des temps modernes, avant le classicisme, gagne à être appréhendée en liaison avec le baroque occitan. Enfin, la littérature des $\mathrm{XIX}^{\mathrm{e}}$ et $\mathrm{XX}^{\mathrm{e}}$ siècle en occitan est suffisamment riche, depuis le temps de Mistral, pour pouvoir être abordée en parallèle avec la littérature française. La diversité des textes d'oc contemporains permet leur insertion dans des groupements de textes thématiques en collège; et l'étude des grands mouvements littéraires, depuis le Romantisme, peut s'enrichir d'un détour par les modalités occitanes de ces mouvements. Par ailleurs, enseigner l'occitan dans une région occitane permet de faire intervenir en classe une dimension rarement accessible au commun des enseignants de lettres, pour des raisons sociologiques évidentes : celle du contact direct avec les auteurs. J'ai eu ainsi l'occasion de faire rencontrer à mes élèves des écrivains d'oc actuels, pour discuter avec eux de leur œuvre, mais aussi de leur genèse, des motivations de celui qui décide un jour qu'il traduira son univers intimes dans les mots de l'occitan. Un tel travail sur les textes intéresse au premier chef les élèves qui ont choisi l'occitan comme langue optionnelle et bien sûr le travail sur la langue, sur les problèmes de traduction en premier lieu, est ici fondamental, et, on s'en doute, hautement formateur. Mais les textes d'oc peuvent aussi être présentés, en traduction, à des élèves qui ne font pas, et ne feront peut-être jamais d'occitan. Et dans ce cas, outre l'enrichissement que le contact avec un texte peut apporter, cette présentation de la littérature d'oc aide aussi l'élève, comme c'était le cas dans le primaire, à se situer dans la région où il vit, en connaissant mieux ce qui a constitué et constitue encore une des dimensions de sa vie culturelle.

A un autre niveau, le professeur d'occitan a forcément reçu, au cours de sa formation, une initiation en matière de romanistique. Et dans le cas de ceux qui ont à enseigner, en plus de l'occitan, une autre langue romane, italien ou espagnol, cette formation peut les aider à éclairer l'enseignement de cette autre langue. C'est là quelque chose que traditionnellement l'enseignement des langues vivantes en France n'est pas habitué à envisager. Rares sont ceux qui peuvent jouer sur la parenté entre les diverses langues romanes, pour mieux montrer que dans une certaine mesure, le monde roman de l'Europe du Sud constitue un continuum. Il y a de cela quelques années, la presse avait signalé avec une sorte d'émerveillement l'expérience menée à Aix-en-Provence d'enseignement combiné de l'italien et de l'espagnol : on est tellement habitué en France à accepter le cloisonnement étanche des diverses matières de l'enseignement secondaire que cet apparentement, considéré ailleurs, en Allemagne par exemple, comme évident, faisait ici figure d'innovation révolutionnaire. Le statut linguistique de l'occitan, et du catalan d'ailleurs, comme langue carrefour de la romanité occidentale lui confère, dans cette perspective, un rôle stratégique considérable, pour peu qu'il soit convenablement exploité. L'apprentissage, on le sait, se construit par comparaison, par la confrontation de savoirs, non par l'opposition de ceux-ci. Dès lors, loin d'enfermer les élèves dans une impasse linguistique, l'enseignement de la langue régionale peut permettre l'ouverture, l'accès à d'autres langues, d'autres cultures. 
24 A un autre niveau, l'occitan peut aider à situer l'espace occitan et son histoire dans l'histoire globale de l'Europe, et de la France au premier chef. Il est régulièrement question, depuis près d'un siècle, d'ouvrir l'enseignement de l'histoire et de la géographie sur la dimension régionale, la plus proche des élèves. L'enseignement de notions de civilisation et d'histoire de l'espace occitan, appuyé sur des textes, a aussi sa place dans l'enseignement de l'histoire et de la géographie en collège et en lycée.

$\mathrm{Au}$ delà de ces dimensions purement disciplinaires, pensées en termes de contenus de savoir, il y en a une autre, incontournable: celle de l'innovation pédagogique. Le seul mérite de la situation de marginalité faite, dans le système scolaire français, aux langues régionales, est qu'elle impose l'inventivité. On ne peut enseigner l'occitan comme une de ces matières qui ont en quelque sorte cours forcé dans les programmes. Vient au cours d'occitan celui qui le veut bien. Et il le voudra bien à condition que ce cours lui apporte quelque chose - et ce quelque chose ne peut pas être simplement un moment de distraction. La classe de langue régionale constitue un de ces lieux de liberté relative qui permettent de faire de l'élève un véritable acteur, dans un projet pédagogique innovant. Mon point de vue sur ce point précis n'est pas simplement du domaine de la proposition théorique. J'ai eu l'occasion, dans mon lycée nîmois, de mener à bien des projets d'édition d'ouvrages contenant des textes d'écrivains occitans, mais aussi des textes d'élèves, sur des thèmes donnés - l'enfance, la ville, les couleurs... textes illustrés par des élèves d'arts appliqués, dans le cadre de projets interdisciplinaires associant plusieurs enseignants et plusieurs classes. De telles expériences montrent concrètement comment l'occitan peut être, s'il est pris au sérieux, le lieu de rencontre dynamique entre des savoirs et des savoirs faire a priori éloignés les uns des autres.

(Philippe MARTEL) Après ces introductions, le débat est ouvert. On ne peut ici le refléter dans toute sa richesse. Quelques indications générales toutefois.

On ne peut manquer d'abord de signaler que l'un des leitmotivs de la discussion a été l'exposé, très concret et malheureusement nourri d'expérience amère, des difficultés pratiques que rencontre celui qui se mêle d'enseigner la langue régionale. Le problème de la formation initiale et permanente est revenu plusieurs fois. Comme celui de la difficulté à trouver des outils pédagogiques adaptés, malgré le travail remarquable accompli par le CRDP de Montpellier, autour notamment de la revue Lenga e païs d'oc. Mais ce travail mériterait d'être poursuivi et mieux diffusé. Si l'enseignant est habitué à bâtir lui-même son corpus documentaire, s'il est souvent, pour peu qu'il ait passé l'âge de la prime jeunesse, un autodidacte en occitan, faute d'avoir pu bénéficier en son temps de l'enseignement qu'il dispense aujourd'hui à ses cadets, il va de soi qu'on ne peut indéfiniment exiger de lui qu'il refasse le monde, à ses frais, au début de chaque année scolaire. L'enseignement de l'occitan et du catalan a besoin d'outils accessibles et, dans la mesure du possible, attrayants et modernes, faisant notamment intervenir les nouvelles technologies. Encore faut-il que les moyens soient là.

Il a été aussi souligné, sur le fond, qu'il y a une autre dimension, indispensable au bon fonctionnement d'une classe : celle du plaisir de la découverte. Certains intervenants, au cours du colloque, ont semblé douter que le plaisir d'apprendre puisse être une valeur de citoyenneté. Nous ne sommes pas d'accord. Le plaisir a son rôle à jouer dans l'apprentissage, comme dans la vie en collectivité. Sauf à rêver d'une citoyenneté austère, à visage de père fouettard... 

comme moyen d'accès à une meilleure intégration dans la société locale. Un témoignage intéressant a été apporté par une enseignante du primaire de la région de Montpellier : elle a pu constater que les élèves, et les parents les plus motivés par l'occitan n'étaient en fait pas originaires de la région. Ils demandaient justement à l'occitan une clé pour pénétrer le milieu où la vie les avait conduits. En revanche, et cela n'étonnera pas ceux qui connaissent la situation sociolinguistique occitane, les «indigènes » avaient parfois du mal à se déprendre de l'attitude traditionnelle de recul face à un idiome traditionnellement dévalorisé par l'institution. Autant dire que contrairement à ce que pourraient croire a priori ceux qui se méfient des langues régionales, elles n'ont nullement vocation évidente à constituer le bunker identitaire de populations de souche désireuses de maintenir la distance avec de nouveaux venus. Oserions-nous dire qu'en fait une certaine forme de défense et illustration de la francité jouerait bien mieux ce rôle de ligne Maginot pour vrais nationaux?

Ce qui nous ramène, pour finir, au problème de la citoyenneté. Nous avons présenté l'enseignement des langues régionales, au delà de l'apport proprement culturel qu'elle constituent, comme lieu de l'ouverture sur la différence, et plus encore - et potentiellement moins dangereux - comme lieu de la confrontation pacifique, de l'échange dialectique entre différences, du dialogue des langues et des cultures. Nous savons bien, d'expérience amère là encore, que de telles affirmations, même nourries d'illustrations concrètes et vécues, risquent de rencontrer un certain scepticisme: la langue qui servait à nos grands pères à parler aux bêtes pourrait donc constituer une porte vers l'universel? Elle ne serait donc pas synonyme de repli sur le terroir? Elle aurait son rôle à jouer dans la formation du citoyen du troisième millénaire ? On aura du mal à nous croire sur parole, et l'on préfèrera peut-être croire que tout ce qui précède relève du credo militant, et de rien d'autre.

Et si l'on prenait le problème par un autre bout? Qu'a apporté à la construction de la citoyenneté l'exclusion séculaire des langues régionales, et qu'apporte aujourd'hui leur marginalisation? Il n'est pas du tout certain que l'unification nationale ne pouvait se faire que par la destruction de tout ce qui n'était pas le français, sauf à penser que l'unité de langue suffit à faire l'unité d'un corps social. A ce compte, il faut croire que Communards et Versaillais, résistants et miliciens parlaient des langues différentes, et qu'ils se sont entretués pour des questions de grammaire. En réalité, non seulement les conflits, graves et nombreux, qui scandent l'histoire de France n'ont jamais été provoqués par la question linguistique, mais encore il n'y a jamais eu dans les provinces périphériques où une langue différente du français est parlée de mouvement de rejet de la France s'appuyant sur l'existence de cette langue. En bref, la pluralité linguistique n'a jamais entravé l'adhésion à la nation française, parce que cette adhésion se fondait sur d'autres motivations, ou allait tout simplement de soi, dans des zones qui en tout état de cause n'avaient guère d'alternative. Et, soit dit en passant, on ne peut simultanément affirmer que le fondement de la citoyenneté en France repose sur le contrat social, et le lien politique entre les citoyens, et affirmer que c'est l'usage exclusif de la langue française qui constitue ce lien, sauf à prétendre que le modèle national français est de type ethnique. Or, on ne cesse de nous répéter le contraire. Il faut choisir.

Nul ne contestera bien sûr, que l'acquisition du français constituait une nécessité. De fait, les populations elles-mêmes l'acceptaient. La question est de savoir si cette acquisition ne pouvait se faire que par la destruction des pratiques linguistiques réelles des citoyens et

Tréma, 15-16 | 1999 
de leurs enfants. Certes, on a longtemps cru en France que le bilinguisme n'était pas possible, que l'enfant ne pouvait parler qu'une langue. C'est l'argument utilisé dans la circulaire de Monzie de 1925, le seul texte officiel français qui ait pris la peine d'argumenter son refus d'accorder la moindre place aux langues régionales. Est-il encore recevable aujourd'hui?

On peut par contre se demander si ce qui fonde le rejet des "patois» depuis l'Ancien Régime, ce n'est pas le mépris, bien peu citoyen, des élites à l'égard d'idiomes qu'elles ne pratiquent pas? Dès lors, ce rejet, et la primauté absolue donnée au français - sous sa forme officielle, sans considération pour ses usages populaires, bien sûr, renvoie moins à une quelconque logique politique ou pédagogique qu'à une logique d'exclusion sociale.

A l'enfant qui arrivait à l'école de la République porteur de ses propres acquis linguistiques, on ne proposait nullement un travail sur ces acquis lui permettant d'acquérir, par la suite, la maîtrise raisonnée du français ; on faisait au contraire comme si cet acquis n'existait pas. Sans se rendre compte que du même coup on illégitimait la langue de la famille, quitte à créer un conflit entre l'écolier et le milieu dont il était issu. Que les populations occitanes, catalanes, bretonnes... aient fini par acquérir le français n'efface pas la blessure intime qu'a constitué la négation de leurs langues propres, et le reniement que l'on exigeait d'elles. Aujourd'hui, la question ne se pose plus dans les mêmes termes pour les populations dont nous venons de parler. Mais elle se pose peutêtre pour les populations issues des immigrations les plus récentes. Il a été question, au cours du colloque, des jeunes des banlieues, et de la déliquescence des structures familiales dans ces banlieues qui expliquait - en partie! leur perte de repères, et leurs attitudes de refus violent de la société. Laissons de côté la question de savoir si c'est vraiment la seule explication. Laissons aussi de côté la question de savoir s'il suffirait que l'école dispensât des cours d'éducation civique et morale à l'ancienne pour que ces comportements déviants disparaissent. Notons simplement qu'il ne faut pas s'étonner de voir rejeter l'autorité de parents qui, souffrant déjà de la dévalorisation sociale qui s'attache aux activités professionnelles qui leur sont laissées, se sont retrouvés au surplus en porte-à-faux total par rapport au modèle culturel que l'école imposait à leurs enfants. La gravité des problèmes qui se posent dans ces banlieues ne doit pas nous faire oublier que l'attitude de l'institution scolaire à l'égard de ceux qui les peuplent n'a été somme toute que la continuation de celle qu'elle avait déjà eue face aux différences internes de la société française à l'époque antérieure.

En bref : non seulement le refus des cultures et des langues différentes n'apporte rien à la citoyenneté, mais, objectivement, il nous semble qu'elle l'affaiblit.

Il faut donc imaginer, et appliquer une autre méthode. Cela implique, de la part des défenseurs des langues minoritaires, le rejet de tout exclusivisme. Ces langues ne doivent pas être des prisons, mais des portes qui ouvrent sur d'autres cultures. L'apprentissage conjoint de deux langues, au delà des profits proprement cognitifs que l'enfant peut en tirer, peut aussi l'éduquer à la coexistence des différences, et à leur complémentarité. L'ensemble des participants à notre atelier ne se reconnait guère dans le culte d'un droit à la différence exclusif de tout ce qui relève de la ressemblance. Ils ne croient pas davantage que les langues régionales n'aient leur place qu'en dehors des programmes et du projet éducatif global de l'école de la République. Ils pensent au contraire que c'est dans le développement d'une conception ouverte de la culture nationale, comme dans le combat pour un décloisonnement et la déhiérarchisation des savoirs que l'occitan, le catalan, le berbère et les autres peuvent jouer leur rôle. Non point celui d'un marqueur 
identitaire qui distingue et sépare, mais celui d'une parole libérée porteuse de dialogue et d'échange.

\section{RÉSUMÉS}

Pourquoi la culture de demain aurait-elle besoin des langues «régionales» pour la construction de la citoyenneté ? Parce que, notamment, elles permettent de lire le milieu où vivent les enfants, ouvrent sur d'autres langues voisines et que leur exercice rompt avec une tradition d'exclusivisme culturel ...

Why might tomorrow's culture have any need for "regional languages" in the fulfilment of citizenship? The reason it will is that, among other reason, they allow one to comprehend the context in which the children live, they broaden one' horizons by being the keys to understanding related languages, and last but not least, because their practice constitutes a breaks with a tradition of cultural exclusion.

INDEX

Mots-clés : citoyenneté européenne, occitan, plurilinguisme

Keywords : european citizenship, occitan language, plurilingualism

\section{AUTEUR}

PHILIPPE MARTEL

CNRS, Université Montpellier III 